\title{
LESS COMMON "DOPING" AGENTS AND SUBSTANCES ENCOUNTERED DURING ROUTINE SCREENING FOR DRUGS
}

\author{
P. E. HAYWOOD, L.R.I.C. and PATRICIA CHALMERS, B.Sc. \\ Racecourse Security Services' Laboratories, P.O. Box 15, Snailwell Road, \\ Newmarket, Suffolk, England, CB8 7DT
}

\section{Introduction}

In the routine analysis of horse body fluids for the presence of dope, substances are occasionally encountered that may give rise to a suspicion of doping. Over a number of years, we have studied several such substances which have occurred in the basic fraction and have effected partial or complete identification in some cases.
\end{abstract}

ABSTRACT

The chromatographic and spectroscopic properties of several unusual substances which have been detected in the "alkaloidal" chloroform extract from racehorse urine and saliva samples are reported. Some of these substances have been identified by combined gas chromatography-mass spectrometry and the source of the substance is stated where this is known. Other substances whose identity is not known have been detected and their mass spectra show characteristic amine fragments. The occurrence of these unidentified substances is more frequent in aged urine samples and it would therefore appear that they are associated with putrefaction.

\section{Materials and Methods}

The compounds reported were isolated by the normal solvent extraction procedure used in this laboratory. This procedure is summarized in Table $\mathrm{I}$.

Chemicals and reagents were all analytical grade. Gas chromatographic conditions are summarized in Table II and paper chromatography was effected by the ascending technique using the butanol-citrate system (Clarke, 1969). Spots were located by examination under ultra violet light (254n.m.) followed by spraying with bromocresol green and iodoplatinate reagents. Mass spectra were obtained using either combined gas chromatography-mass spectrometry or the direct insertion probe technique, using an AEI MS20 mass spectrometer.

\section{Results}

The substances that have been investigated are listed in Table III together with their spectroscopic and chromatographic properties.

\section{Discussion}

The majority of these substances were initially detected by routine gas chromatography using system a. Dicyclohexylamine and cresol were identified using combined gas chromatography-mass spectrometry by refer- ence to the eight peak index (Mass Spectrometry Data Centre -1970 , a \& b). The order of intensity of peaks in the cresol spectrum indicated that it was predominantly the p-isomer. The extractability of cresol, a weak acid, into the basic fraction was confirmed using a sample of urine at $\mathrm{pH} 9.5$ to which cresol had been added. The fact that cresol has a pK value of 10 would probably explain these findings (Hodgman, 1962).

The presence of p-cresol has previously been reported in hydrolysed urine from both mares (Marshall 1937), and stallions (Campbell and Hey 1944), when it was observed that increases in the concentration of p-cresol were accompanied by corresponding increases in the concentration of oestrogenic hormones.

Methyl palmitate is frequently detected by routine gas chromatography. Its identity was established by comparison of its mass spectrum with that of an authentic sample. There is evidence to suggest that it occurs by contamination of the extract with soap or cosmetics, methyl palmitate having been detected in the soap used in this laboratory. It is not therefore considered to be indicative of doping and we refer to its detection as a false positive result. Also in this category are two unknown substances whose mass spectra are given in figure 1. They show characteristic amine fragments with mass to charge ratios of 84 (substance No. 1) and 58 and 86 (substance No. 2) respectively. Possible structures for these fragments are shown in figure 2. The occurrence of these two compounds is more frequent in aged urine samples and it seems likely that they are associated with putrefaction.

The phthalate esters were detected during the gas chromatographic analysis of a sample suspected of containing procaine. The chromatogram showed five peaks, one of which was identified as procaine by combined gas chromatography-mass spectrometry. The other four peaks were identified as phthalate esters by reference to 
TABLE I

URINE (120 m/s)

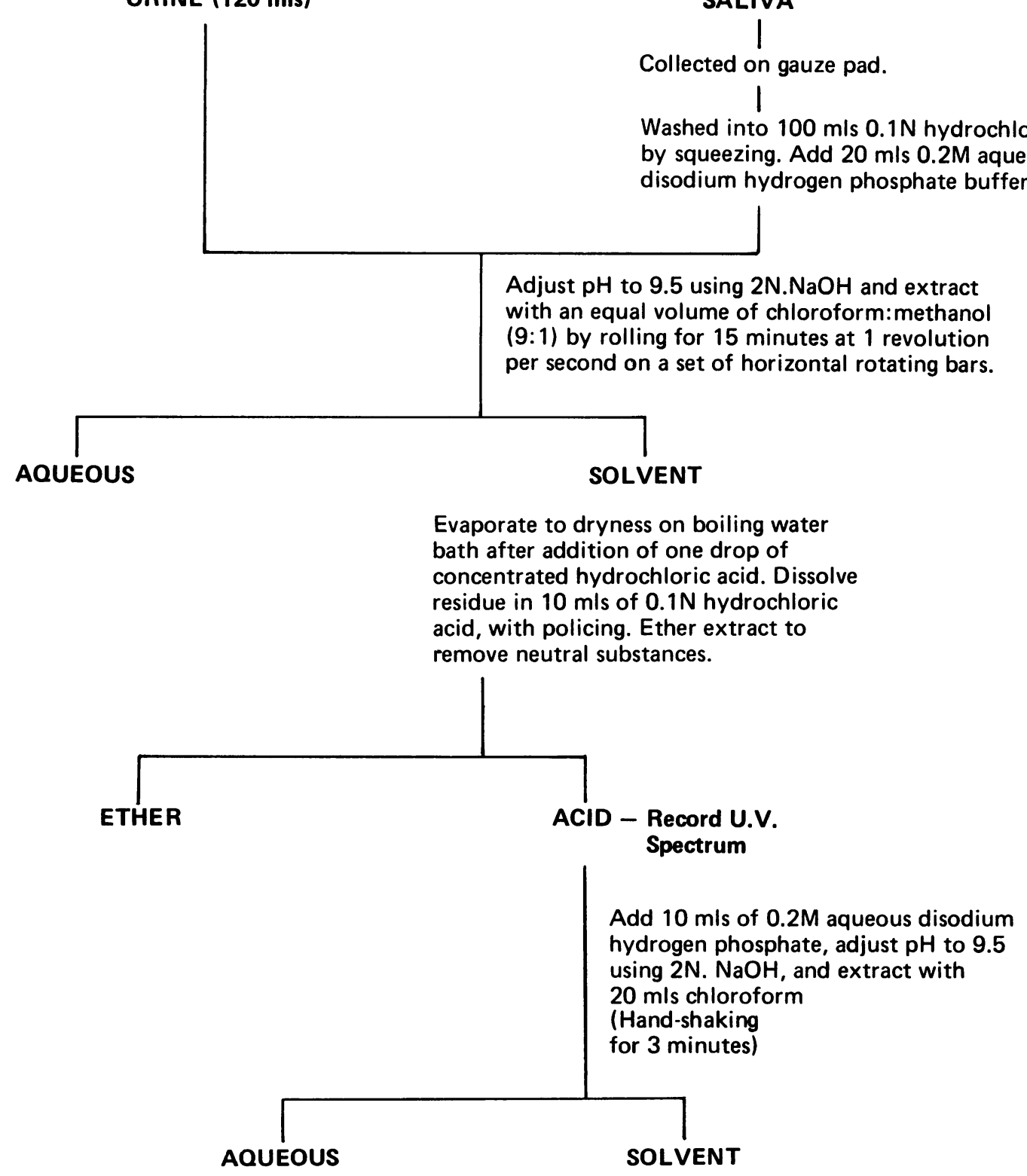

by squeezing. Add $20 \mathrm{mls} 0.2 \mathrm{M}$ aqueous

Adjust $\mathrm{pH}$ to 9.5 using $2 \mathrm{~N} . \mathrm{NaOH}$ and extract with an equal volume of chloroform:methanol (9:1) by rolling for 15 minutes at 1 revolution per second on a set of horizontal rotating bars.

Evaporate to dryness on boiling water bath after addition of one drop of

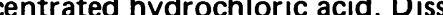
residue in $10 \mathrm{mls}$ of $0.1 \mathrm{~N}$ hydrochloric acid, with policing. Ether extract to move neutral substances.

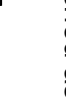

Evaporate to dryness after addition of 1 drop concentrated hydrochloric acid

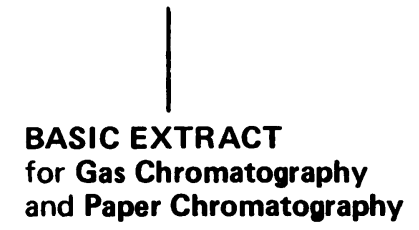


TABLE II

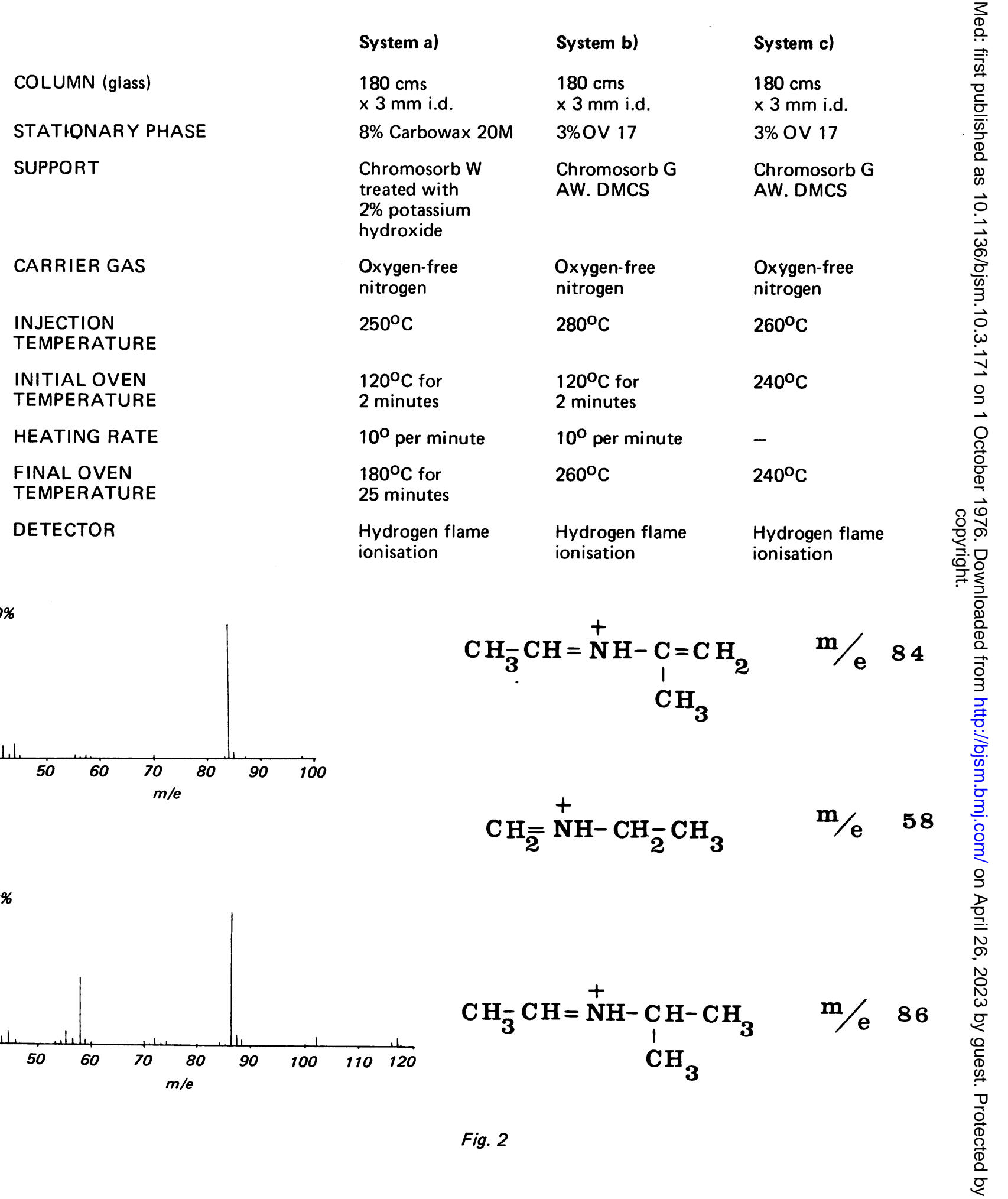




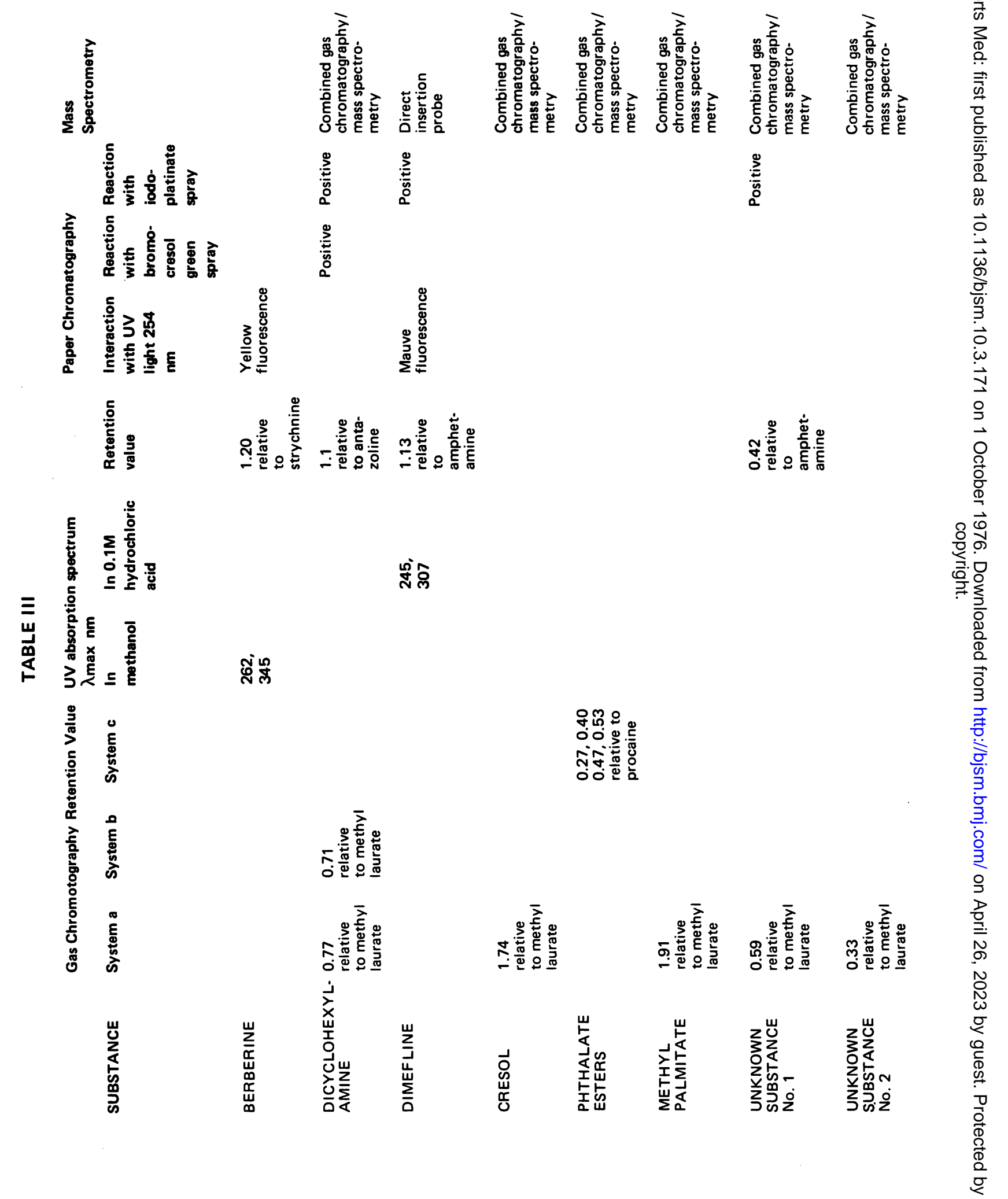


the eight peak index, their spectra showing a base peak at $\mathrm{m} / \mathrm{e} 149$ which is characteristic of many phthalate esters (Mass Spectrometry Data Centre - 1970, c). There is no evidence to suggest that the occurrence of phthalate esters was connected with the presence of procaine.

Berberine (figure 3) and dimefline (figure 4) were detected initially by ultra violet spectrophotometry and paper chromatography. The presence of berberine,

$\Gamma$<smiles></smiles>
$\mathrm{OH}-$

Fig. 3<smiles>CCN(C)Cc1c(OC)ccc2c(=O)c(C)c(-c3ccccc3)oc12</smiles>

Fig. 4

which occurred in a sample of saliva, was confirmed by spectrophotofluorimetry, the optimum activation wavelength of 347 nanometres yielding maximum fluorescence at 525 nanometres in ethanolic solution. Activation and fluorescence spectra are shown in figure 5.

Subsequent enquiries revealed that a styptic mixture had been administered as a drench to prevent epistaxis. This mixture was said to contain tincture of Hydrastis canadensis (Golden seal) which contains berberine. Berberine was in fact identified in this preparation.

The identity of dimefline, a central nervous system stimulant, was established by mass spectrometry. It is of

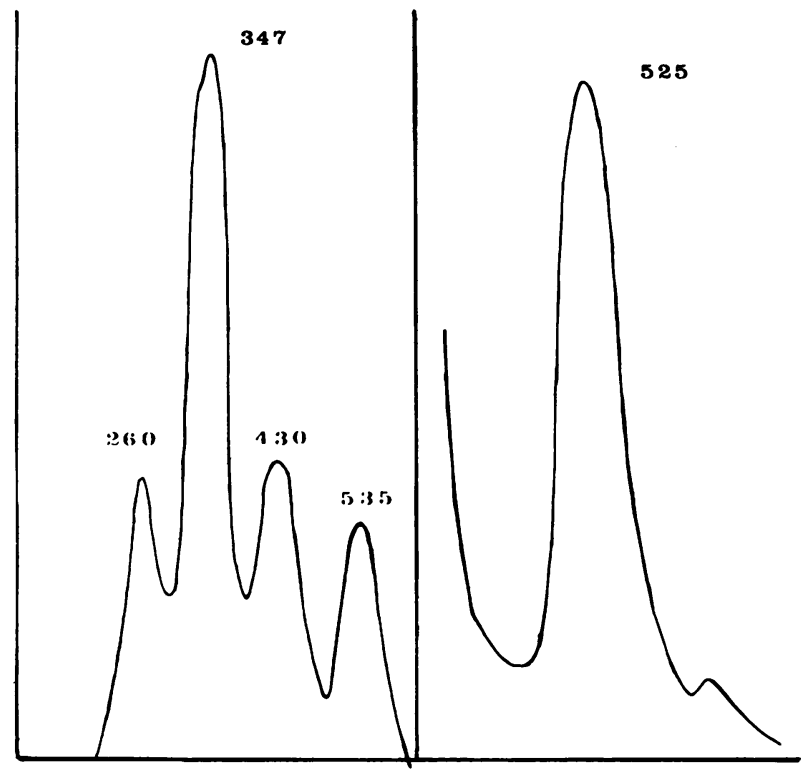

Wavelength in nanometres

Fig. 5

interest to note that when subjected to paper chromatography the extract from the urine sample showed an area of pale green fluorescence under ultra violet light, whereas an authentic sample of dimefline showed an area of mauve fluorescence at the same retention value. Both areas gave a positive reaction with iodoplatinate reagent. Identical spectra were observed when these two areas were eluted for mass spectrometry and it is likely that the pale green fluorescence was due to co-extracted material masking the genuine mauve fluorescence of dimefline. In connection with this case, a tablet extract was submitted for analysis and its properties were consistent with those of the authentic sample of dimefline.

\section{Acknowledgements}

The authors are grateful to Mr. G. A. Oxley for obtaining the mass spectra, and to Dr. E. Houghton for their interpretation. 


\section{REFERENCES}

Clarke, E. G. C. (ed.) 1969. Isolation and identification of drugs, page 34. Pharmaceutical Press, London.

Mass Spectrometry Data Centre, 1970. Eight peak index of mass spectra, 1st edition, vol. 2, table 3, (a) D 0904 page 1197, (b) X 0460 page 1094, (c) Pages 1228-1229, AWRE, Aldermaston, Reading, U.K.

Hodgman, C. D. (ed.) 1962. Handbook of chemistry and physics, 44th edition, page 1754. Chemical Rubber Publishing Company, Cleveland, Ohio, U.S.A.

Marshall, P. G., 1937. Nature London 140, 362.

Campbell, N. R. and Hey, D. H. 1944. Nature London 153, 745.

\section{DISCUSSION}

DELBEKE: Is the extraction procedure valid for amphetamine-like substances? Have you any idea about the analysis of amphetamine-like compounds due to the solubility of the hydrochloride in ether?

HAYWOOD: The extraction procedure is used to detect amphetamine-like substances. This method will detect 10 micrograms of amphetamine added to 100 millilitres of urine.

MAYNARD: We ran into a great number of pyrocresols in urine samples, these are depressed by the use of mercuric chloride in the urine sample. Our samples are sometimes as much as 48 hours in transportation and temperatures in some areas of Canada during the Summer ranges between 90 and $95^{\circ} \mathrm{F}$. Therefore we are getting degradation of the urines and this has been attributed mainly to the fact that stallion urines give the greatest amount of pyrocresol, geldings next and mares least. We do find two other sources of phenolics, one of course is antiseptics, used in washing down and getting into the urine sample. The other is cough medicines using guaiachol, although you can small guaiachol if it is there.

HAYWOOD: In fact we have only detected cresol once and the sample was from Africa.

MAYNARD: This could quite possibly be a high temperature degradation product.

CLARKE: Some 20 years ago I received a sample of urine from Trinidad which had travelled by sea, there was so much phenolic acid in it the specimen resembled a sample of tar.

CHAPMAN: I would like to comment that some years ago we did extract phthalates from horse urine and we got the impression these were probably from the bottles we collected the samples in since the horses were under our own control. In this case it was dibutyl phthalate and we confirmed it by mass spectrometry. It was in the acidic fraction extracted with ethyl acetate, but it was quite a regular contaminant.

JOHNSTON (Canada): Probably the largest number of times that something comes up on the mis-match scan of our GC/MS it is butyl phthalate. It is always there in some quantity and we use plastic containers.

MAYNARD: I would like to suggest in some future meeting a session should be devoted to extraneous substances because we have quite a long list of extraneous substances that have been extracted in the three official laboratories in Canada. Some have been definitely identified, some we don't know what they are although we hope to use the mass spectrometer to identify these too.

TOBIN: Does your Racing Authority take action when you report phthalates?

HAYWOOD: No, I did not report the phthalate, it was really the subject of a procaine enquiry and there seems to be a perfectly rational explanation for its presence. 